\title{
Silicon Tracking System of the future CBM experiment at FAIR
}

\author{
Maksym Teklishyn*i \\ FAIR (Darmstadt, Germany), KINR (Kyiv, Ukraine) \\ E-mail: m.teklishynegsi.de
}

\begin{abstract}
The physics aim of the Compressed Baryonic Matter (CBM) experiment is to explore the phase diagram of strongly interacting matter at highest net baryon densities and moderate temperatures in the range reachable with AA collisions between $2-45 A \mathrm{GeV}$, initially $2-14 A \mathrm{GeV}$ (SIS 100 ). The Silicon Tracking System (STS) is the central detector for charged particle tracking and momentum determination. It is designed to be operated at high occupancy at collision rates up to $10 \mathrm{MHz}$. It is planned to reach the track reconstruction efficiency of $95 \%$. The momentum resolution is expected to be around $1.5 \%$. To achieve these goals, double-sided double-metal silicon sensors with a pitch of $58 \mu \mathrm{m}$ are employed. The sensors are mounted on light-weight carbon ladders, forming 8 tracking stations. The read-out electronics is kept outside of the detector acceptance. The self-triggering read-out electronics is connected with the silicon micro-strip sensors through the multi-line micro-cables. The resulting material budget is about $1 \% X_{0}$ per station. The entire system is going to be operated in a thermal enclosure to maintain constant temperature below $-5^{\circ} \mathrm{C}$.

Before the mass production of the silicon sensors, several studies are performed with prototypes, such as electrical and optical inspection, measurements with the radioactive sources and beam tests of the sensors and the read-out electronics. Particularly, we study the possible impact of the severe radiation environment $\left(10^{14} 1 \mathrm{MeV} \mathrm{n}_{\mathrm{eq}} \mathrm{cm}^{-2}\right)$ to the sensor performance.
\end{abstract}

The STS project is realised in cooperation of institutes from Germany, Poland, Russia and Ukraine. This presentation is given on behalf of the CBM Collaboration.

55th International Winter Meeting on Nuclear Physics

23-27 January, 2017

Bormio, Italy

\footnotetext{
*Speaker.

${ }^{\dagger}$ On behalf of the CBM Collaboration.
} 


\section{The CBM experiment at FAIR}

The aim of the Compressed Baryonic Matter (CBM) experiment is to explore the QCD phase diagram in the region of the high net baryon density. For this purpose both the studies of the collective effects and rare observables are going to be involved. Particularly, the physics program of CBM will concentrate on the measurements of the differential cross-sections of the strange hadrons, such as $K^{+}, \Lambda, \Sigma^{ \pm, 0}, \Xi^{-, 0}, \Omega^{-}$and their resonant states, see Fig.1 (left). In order to collect sufficient number of events one should involve intensive beams that will lead to interaction rates up to $10 \mathrm{MHz}$. It requires fast and radiation-tolerant detection systems as well as reliable free-streaming read-out electronics. [1]

In Fig.1 (right) one can see an interaction rate and a collision energy of the CBM experiment in comparison with the existing and future facilities. The CBM experiment gives a unique possibility to approach the investigation of the QCD phase diagram at high net baryon densities and moderate temperatures.
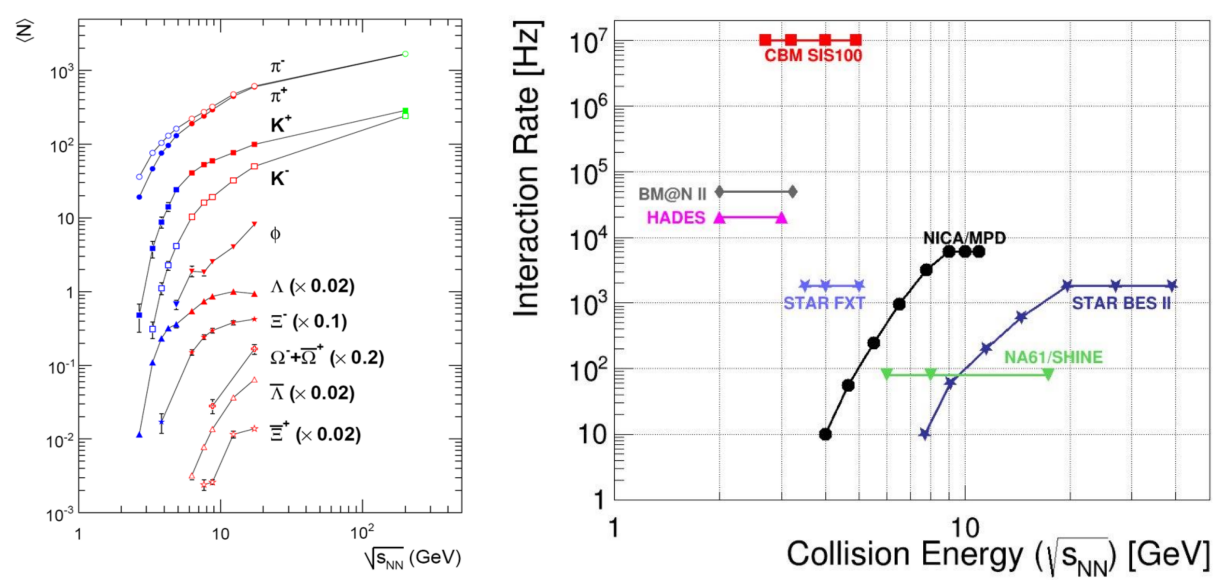

Figure 1: Hadron yields in gold-gold collisions as a function of the centre-of-mass energy for CBM (left) and the interaction rate as a function of the centre-of-mass energy for different experiments (right). [2]

Beside the rare particles, physics program of the CBM experiment includes studies of the collective effects, particularly fluctuations of the hadron flow caused by the phase transition. Dileptons, open charm and charmonium production are also covered by the CBM physics program. [3]

The CBM experiment is foreseen as a part of the future Facility for Antiproton and Ion Research (FAIR) in Darmstadt, Germany. The SIS-100 accelerator will provide high intensity heavy ion beams with the kinetic energy $2-10 \mathrm{AGeV}$ for gold ions and up to $30 \mathrm{GeV}$ for protons.

\section{Concept of the Silicon Tracking System}

The Silicon Tracking System is a core detector of the CBM experiment. It will be placed inside the $1 \mathrm{Tm}$ dipole magnet $30 \mathrm{~cm}$ downstream to the target. The STS detector is dedicated to the tracking of the charged particles and measuring their momenta. The designed angular acceptance of STS is $2.5^{\circ} \leq \theta \leq 25.0^{\circ}$, which is sufficient to track the majority of particles coming from the primary vertex. 
The detection system involves the $300 \mu \mathrm{m}$ thick double-sided double-metal silicon micro-strip sensors of various sizes: $22 \times 62,42 \times 62,62 \times 62$ and $124 \times 62 \mathrm{~mm}^{2}$. Basing on the simulations, the pitch of $58 \mu \mathrm{m}$ was chosen for the best performance. Each side of the sensor contains 1024 strips.

The read-out electronics will be placed outside of the detector acceptance in order to decrease the amount of material and to avoid the severe radiation. It will be connected to the sensors via ultra-thin micro-cables. The $\mathrm{CO}_{2}$ bi-phase cooling system is being developed to deal with the heat produced by the electronics. STS will be placed inside of a thermal enclosure in order to maintain a constant temperature below $-5^{\circ} \mathrm{C}$. It will be vital for the heavily irradiated silicon sensors due to significant increase of the dark current with the absorbed dose.

The radiation environment is expected to be hard due to the nature of the ion-ion interaction. Using FLUKA simulation, the lifetime dose of STS was estimated as $10^{14} 1 \mathrm{MeV} \mathrm{n}_{\mathrm{eq}} \mathrm{cm}^{-2}$ [1].

Silicon sensors will be fixed on the carbon ladders which then will be mounted on the aluminium frame making structures called STS units as it is shown in Fig.2. The units will be organised in the 8 tracking stations with approximately $10 \mathrm{~cm}$ distance between the neighbouring stations.
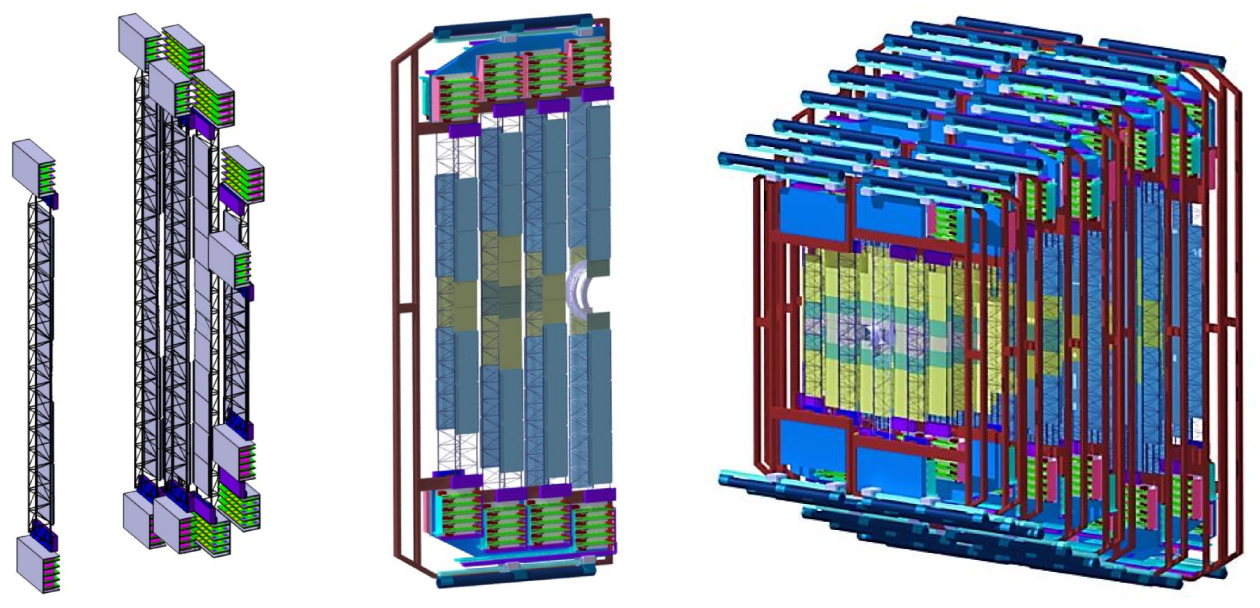

Figure 2: Drawings left to right: carbon ladders, equipped with sensors and read-out electronics, STS unit on the supporting frame and all 8 stations arranged together.

The following key features of the setup are dictated by the physics program of the experiment. At first, STS is foreseen as a tracking detector with an ultra-low material budget: the whole setup, including silicon sensors, carbon supporting structures and read-out cables, contributes no more than than $8 \% X_{0}[1]$. This is necessary for the suppression of the multiple scattering for the lowmomentum particles, which ameliorates position resolution of the interaction points. The designed momentum resolution depends on the particle momentum and in average is about $\Delta p / p \approx 1.5 \%$.

Secobdly, STS can be operated at high occupancy which may reach $\geq 10 \mathrm{MHz} / \mathrm{cm}^{2}$ in the innermost region for the gold-gold central collisions with the beam energy of $10 \mathrm{AGeV}$. Running in the self-triggering mode, each individual STS channel will provide a time resolution of about $5 \mathrm{~ns}$. $[1,2]$ 


\section{Development and quality assurance of the silicon sensors}

\subsection{Layout of the silicon micro-strip sensors}

The double-sided double-metal micro-strip sensors are the building bricks of STS. They come in various sizes, enlarging from the innermost to the outermost regions to fit the detector occupancy. The photo of the silicon sensor prototypes in four different form factors is given in Fig.3, left.
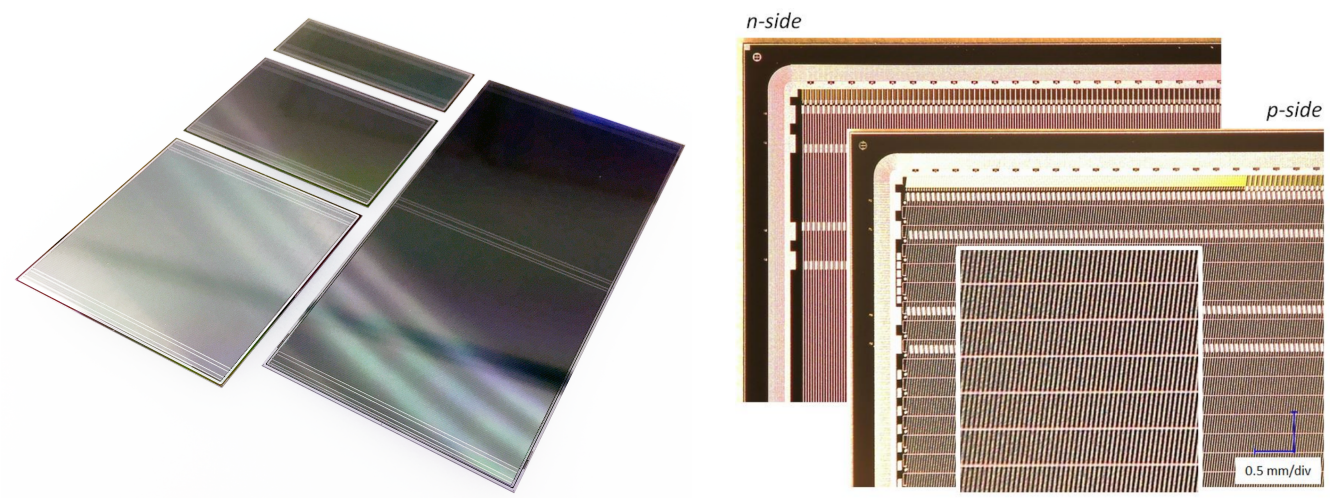

Figure 3: Left: a photo of the silicon micro-strip sensors in four different sizes. Right: microscope photo of the corner of a sensor; pattern of the strips and the connection pads is visible. [2]

The strip pattern on both sides of the different sensors is similar: there are 1024 strips with a pitch of $58 \mu \mathrm{m}$. However, strips on the p-side are inclined by $7.5^{\circ}$ to the edge of the sensor (see Fig.3 right). This way one can have a sufficient position resolution in the horizontal direction (bending plane of the dipole magnet) at the same time suppressing the number of fake interaction points. Short strips on the p-side are interconnected via the second metallisation layer with the corresponding strips on the opposite edge forming a z-like pattern. This is done in order to read the sensor out from one side; however for the testing purposes connecting pads are foreseen both on the top and the bottom edge of the sensor.[1]

Collection of the charge induced by the ionisation radiation inside the sensor sensitive medium is performed via the AC coupling. The dedicated STS-XYTER ASIC [4] is being developed for the silicon sensor read-out. At the moment some limited amount of the chips were produced to be electronically tested before being involved in the read-out chain.

\subsection{Optical inspection}

In the mass production phase more then one thousand of sensors will be created. In order to perform the quality assurance (QA) of such amount of items a set of automatized procedures should be developed. Optical inspection is one of the tests allowing to conclude upon the quality of the item with no risk of damaging it.

The optical test station is being developed by the STS group in Tübingen University. It consists of an XY movable platform with a replaceable vacuum chuck mount. Currently a custom-built three-zone chuck is used for the sensors and a porous aluminium chuck is used for the microcable inspection. A microscope camera and an optical system with a motorised focusing and zoom are placed on the vertical stage. The XY-platform allows to inspect sensors up to a size 
of $124 \times 62 \mathrm{~mm}^{2}$. A flexible software, which allows an adaptation for other hardware, as well as the inspection of various objects, like detector modules, was developed in a frame of this project. [2]

\subsection{Electrical tests}

During the production of sensor prototypes $100 \%$ of the items are tested in the Quality Assurance Centres. However during the mass production stage only $5-10 \%$ of the sensors will pass some set of general tests. Circa of $1 \%$ of the sensors will be deeply studied in order to verify the QA results provided by the sensor manufacturers.

The setup for the sensor characterisation must be installed in a clean room with the temperature and humidity control as many of the tested characteristics, especially current, express significant dependence on the environment conditions. For example, the leakage current after the measurement should be normalised to the nominal room temperature using relation:

$$
I \propto T^{2} e^{-\frac{E_{g}}{2 k_{B} T}},
$$

where $E_{g}=1.12 \mathrm{eV}$ is the band-gap width at room temperature and $k_{B}=8.62 \mathrm{eV} / \mathrm{K}$ is a Boltzmann constant. [6]

Several quantities, such as the total capacitance of the sensor, leakage current, individual current and coupling capacitance of the strips, can be measured involving probe stations in GSI, Darmstadt and Tübingen University.

\subsection{Charge collection studies with the $\beta$-source}
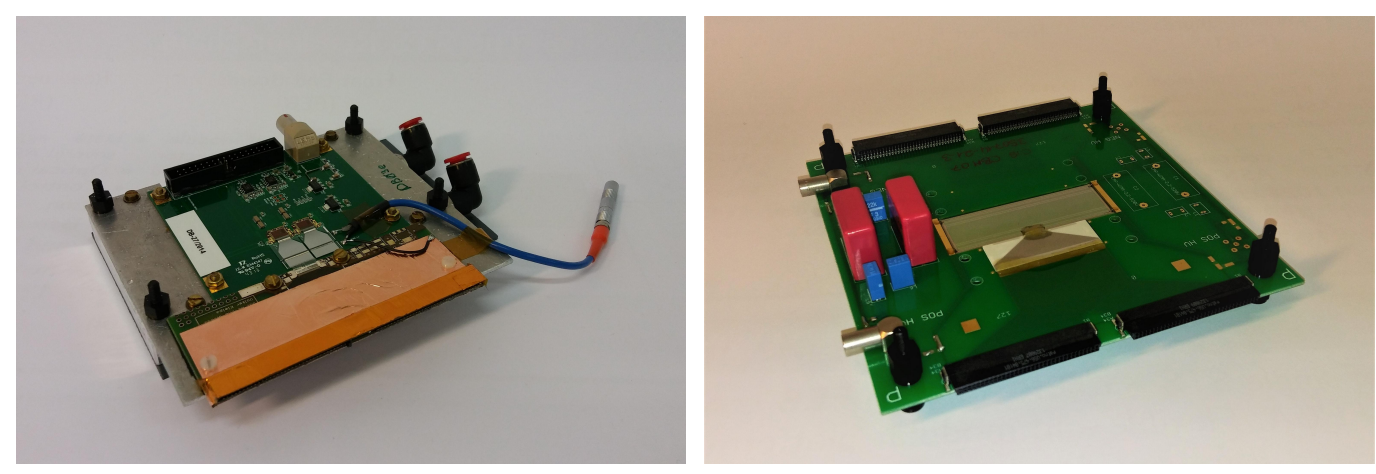

Figure 4: The Alibava front-end board with two 64-pin ERNI connectors mounted on the aluminium cooling block (left). The $62 \times 22 \mathrm{~mm}^{2}$ sensor in the supporting PCB.

The main property of the silicon sensors is an ability to transform an electrical charge induced by the charged particle in the sensitive medium to the current pulse that can be read out by the preamplifier. The ratio of the electron-hole pairs, exited by the ionisation radiation to the number of charge carriers detected by the front-end electronics is commonly called the charge collection efficiency $\varepsilon=Q_{\mathrm{r} / \mathrm{o}} / Q_{\mathrm{i}}$. The value of the $\varepsilon$ can be measured in the laboratory using a source of $\beta$-radiation, such as ${ }^{90} \mathrm{Sr}$ isotope. The STS-XYTER chip is currently an object of tests and may not 
be used to study properties of the silicon micro-strip sensors. For such needs we involve electronics based on the Beetle chip [5], initially designed for the tracking system of the LHCb experiment.

The Beetle chip is operated as an integrated part of the Alibava system. It allows to simultaneously measure up to 256 channels from the one side of the sensor. The front end board of the Alibava system was deeply customised in order to read out the large set of various sensors. The fan-out was wire-bonded with two 64-pin ERNI connectors to the pitch adaptor of the Beetle chip. The modified front-end board is shown in Fig.4, left, and the $62 \times 22 \mathrm{~mm}^{2}$ sensor attached to the pitch adaptor on the supporting frame is shown in Fig.4, right.

To study the response of the sensor to the electrons of the ${ }^{90} \mathrm{Sr}$ source the following setup was developed. A set of aligned aluminium collimators holds the supporting PCB with a sensor glued in. The plastic scintillator with an attached PMT is paced beneath the sensor; it triggers the read-out electronics when an energetic electron passes through the sensor sensitive area. The energy deposited by a charged particle in thin layer of silicon obeys Landau-Vavilov distribution and depends on the particle energy:

$$
\varphi(E, \xi) \otimes e^{-\frac{E^{2}}{2 \delta_{0}^{2}}},
$$

where $\varphi$ is the Landau distribution, $E$ is a kinetic energy of the particle, $\xi$ is a characteristic energy of the Landau distribution and $\delta_{0}^{2}$ represents additional smearing due to the electron-shell interaction [7]. With the sufficiently high momentum $(\gamma \gtrsim 2)$ the mean energy deposited in the material almost does not depend on the particle kinetic energy. Particularly, for electrons $\langle\Delta E\rangle$ varies less than $8 \%$ in a range $1.5 \leq E_{k} \leq 2.5 \mathrm{MeV}$. Thus one can use a hard component of the ${ }^{90} \mathrm{Y}$ electron spectrum (decay product of ${ }^{90} \mathrm{Sr}$ ) by selecting the most energetic electrons, registered in the scintillating crystal. Examples of the Landau-like spectra acquired with $62 \times 62 \mathrm{~mm}^{2}$ sensor are given in Fig.5.
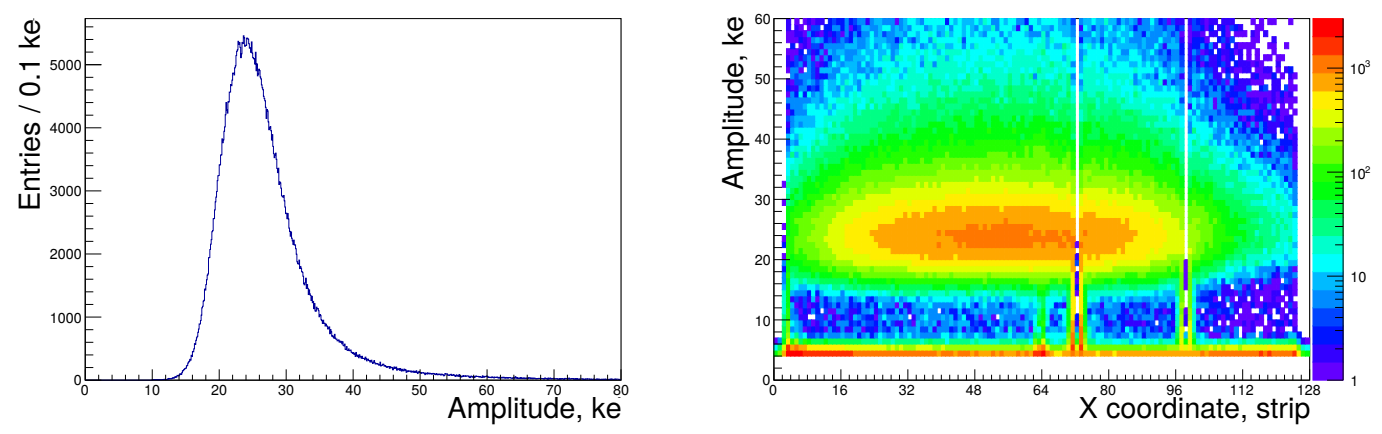

Figure 5: The spectrum of the charge in kilo-electrons, registered by the read-out electronics, attached to central 128 strips of the $62 \times 62 \mathrm{~mm}^{2}$ silicon sensor (left). Charge-position distribution for the same setup: a $\beta$-source exposures mostly the central strips of the sensitive area (right).

One of the available front-end boards was directly wire-bonded to the $62 \times 62 \mathrm{~mm}^{2}$ singlemetal sensor prototype to investigate on the alternative connection schemes. Three possible connection schemes are represented in Fig.6. The 256 read-out channels were divided in three groups: 
128 channels were connected one-by-one to the sensor strips, the rest were equally divided between two alternative connections: two-to-one and every second strip read-out.

This investigation was triggered by considering particles, interacting with the outermost part of STS: in such a case they cross the sensor surface at an angle $\geq 20^{\circ}$, sharing the charge between several strips. Potentially it can affect the signal-to-noise ratio, degrading the tracking performance. Merging strips in pairs could help to overcome this issue.

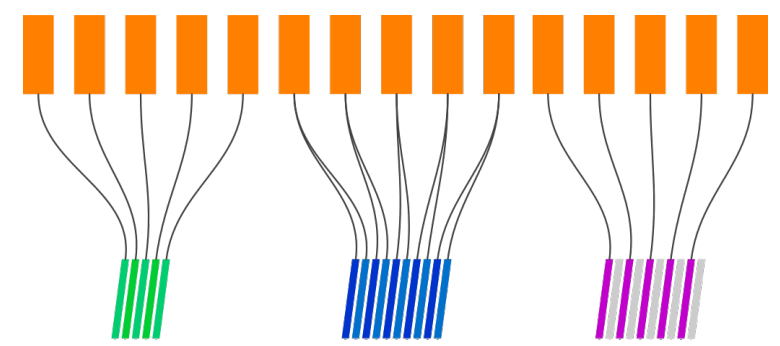

Figure 6: Schematic of the alternative ways to connect read-out electronic to the sensor (left to right): every strip connected, two strips attached to one read-out channel, every second strip connected.

The results of these studies are shown in Fig.7. As one can see, two-to-one connection allows to collect more charge, however the noise level increases due to a higher captive load. Connection scheme with every second channel read-out demonstrates the opposite trend.
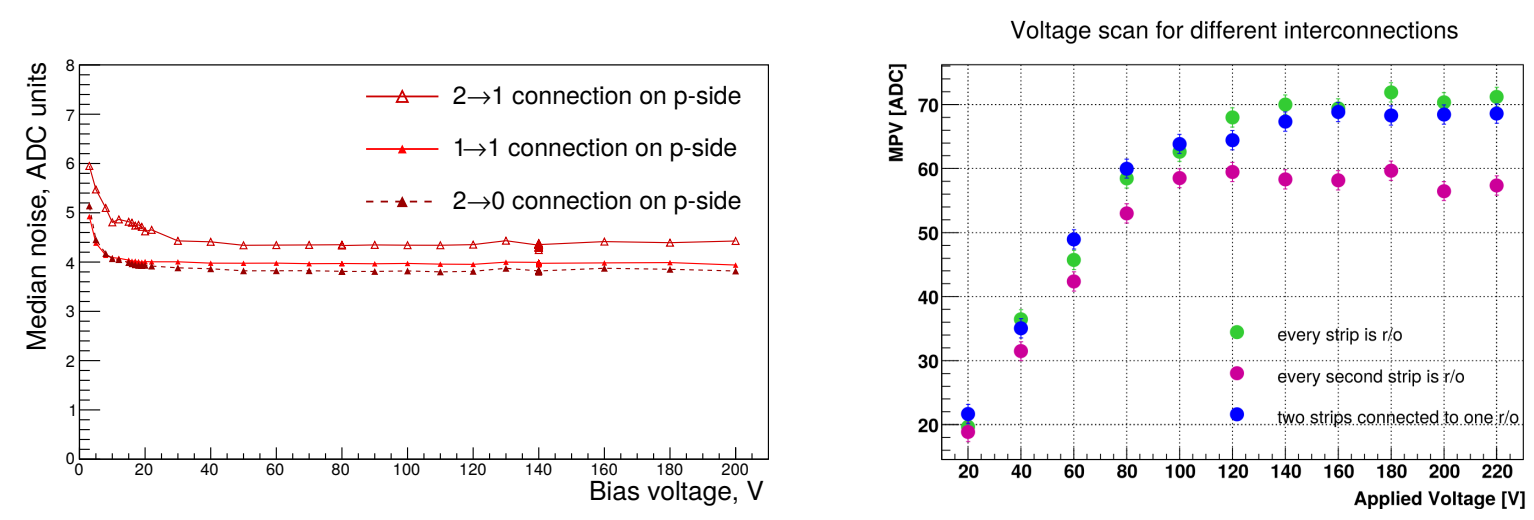

Figure 7: Dependence of the noise (left) and the signal mode (right) [2] on the bias voltage for the different connection schemes.

Further investigation is required in order to make a conclusion concerning alternative connection schemes. Measurements with particles with the non-zero incident angle were held and the resulting data are being analysed.

\section{System integration}

Currently the main activities on the system integration are concentrated on the development of the techniques of component assembling, cooling and cabling. Dummy modules for one detector unit were assembled in order to obtain an experience with the longest micro-cables and the largest sensors $\left(124 \times 62 \mathrm{~mm}^{2}\right)$. All necessary tools have been modified in order to handle the latest sensor 
prototypes. In Fig. 8 one can see examples of the assembled dummy modules with stacks of microcables attached to the read-out chips.

Meanwhile, significant effort is invested into the development of the mechanical support for the sensors and the read-out electronics. The prototype representing one-quarter on the largest station has been made. It allows to study possible mechanical issues before going to the full-scale setup. Precise mechanical positioning is highly important for STS since even small misalignment may significantly affect tracking performance. [2]
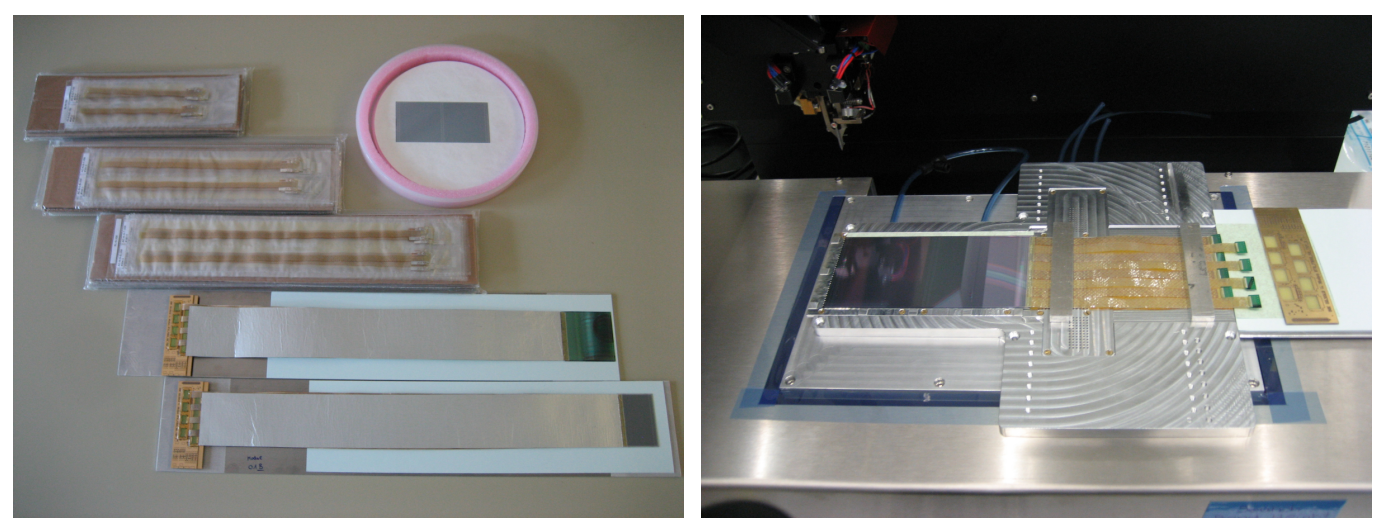

Figure 8: Dummy-modules (left) and TAB-bonding on a $124 \mathrm{~mm}$ sensor (right). [2]

\section{Conclusions}

There are many activities held by the STS work group of the CBM collaboration. The projects are developed by the international participants from several institutions in Germany, Russia, Poland and Ukraine. An important milestone coming in 2017 is the sensor production readiness, which means freezing the design of the silicon sensors and starting their mass production for the final detector. The STS construction phase is then foreseen until 2021.

\section{References}

[1] J. Heuser et al. Darmstadt, GSI, GSI-2013-05499, GSI Report 2013-4, 167 p., 2013

[2] I. Selyuzhenkov, A. Toia, CBM Progress Report 2016, Darmstadt, GSI, GSI-2017-00564, 2017

[3] B. Friman, C. Hohne, J. Knoll, S. Leupold, J. Randrup, R. Rapp and P. Senger, Lect. Notes Phys. 814 (2011) pp.1.

[4] K. Kasinski, R. Kleczek and R. Szczygiel, JINST 11 (2016) no.02, C02024.

[5] S. Löchner and M. Schmelling, LHCb-2005-105, CERN-LHCb-2005-105.

[6] O. Bertini et al., "Specifications for Quality Assurance of Microstrip Sensors in the CBM Silicon Tracking System”, CBM-STS Note 2016-1

[7] H. Bichsel, Rev. Mod. Phys. 60 (1988) 663. doi:10.1103/RevModPhys.60.663 